\title{
Realimentação derivativa e modelo fuzzy Takagi-Sugeno para controle da articulação do joelho de pacientes paraplégicos com o uso de acelerômetros
}

\author{
Ruberlei Gaino, Marcelo Carvalho Minhoto Teixeira, Aparecido Augusto de Carvalho*, \\ Edvaldo Assunção, Rodrigo Cardim, Marcelo Augusto Assunção Sanches, Márcio Roberto Covacic
}

Resumo Sistemas de controle em malha fechada têm sido utilizados para movimentar músculos e articulações dos membros inferiores de pacientes paraplégicos, por meio de estimulação elétrica funcional (Functional Electrical Stimulation - FES). Com uso de um neuroestimulador para aplicar pulsos no músculo quadríceps, o sinal de realimentação relacionado ao ângulo da articulação do joelho pode ser obtido utilizando-se um eletrogoniômetro. No entanto, a utilização de um acelerômetro facilita as medições devido ao seu menor tamanho e maior facilidade de fixação sobre a pele do paciente. Neste trabalho é apresentado um novo método de projeto de controle, visando variar o ângulo da articulação do joelho em pacientes paraplégicos por meio de estimulação elétrica funcional, sendo apresentada uma simulação com valores de parâmetros disponíveis na literatura. O sistema de controle não-linear da dinâmica do paciente paraplégico é descrito por modelos fuzzy Takagi-Sugeno e o sinal de realimentação é obtido por meio de acelerômetros. O projeto considerou todas as não-linearidades da planta e satisfez às especificações mais importantes, tais como a estabilidade segundo Lyapunov, a taxa de decaimento e a restrição no sinal da entrada. Os resultados obtidos na simulação do sistema de controle proposto, com o ângulo da articulação do joelho variando na faixa de $0^{\circ}$ a $30^{\circ}$, foram satisfatórios. O sistema de controle proposto é viável, fornecendo uma nova alternativa para o controle do ângulo da articulação do joelho, utilizando sensores mais leves e confortáveis para os pacientes.

Palavras-chave Sensor de aceleração, estimulação elétrica funcional, controle não-linear, modelo fuzzy Takagi-Sugeno, lesão medular, movimentos funcionais.

\section{Derivative feedback and Takagi-Sugeno fuzzy model for knee joint angle control of paraplegic patients using accelerometers}

\footnotetext{
Abstract Feedback control systems have been used to move the muscles and joints of the limbs of paraplegic patients. The feedback signal, related to the knee joint angle, can be obtained by using an electrogoniometer. However, the use of accelerometers can help the measurements due the facility of adhering these devices to the skin. Accelerometers are also very suitable for these applications due their small dimensions and weight. In this paper a new method for designing a control system that can vary the knee joint angle using Functional Electrical Stimulation (FES) is presented, as well as a simulation with parameters values available in the literature. The nonlinear control system was represented by a Takagi-Sugeno fuzzy model and the feedback signals were obtained by using accelerometers. The design method considered all plant nonlinearities and was efficient and reliable to control the leg position of a paraplegic patient with the angle of the knee ranging from $0^{\circ}$ to $30^{\circ}$, considering electric stimulation at the quadriceps muscle. The proposed method is viable and offers a new alternative for designing control systems of the knee joint angle using more comfortable sensors for the patients.
}

Keywords Accelerometers, functional electrical stimulation, nonlinear control, Takagi-Sugeno fuzzy models, marrow lesion, functional movements. 


\section{Extended Abstract}

\section{Introduction}

The Functional Electrical Stimulation (FES) has been used to move muscles and joints of the limbs on paraplegic, quadriplegic and hemiplegic patients. There are several FES studies, including different muscle and knee joint mathematical models, regarding the movement control of paraplegic patients.

In Gaino (2009) and Teixeira et al. (2006a,b), studies and simulations of a paraplegic's leg position control were performed for the first time using Takagi-Sugeno (T-S) fuzzy models (Takagi and Sugeno, 1985; Tanaka et al., 1998; Teixeira and Żak, 1999). The leg mathematical model, proposed by Ferrarin and Pedotti (2000), was adopted to obtain the relationship between the muscle torque with the pulse width of the electrical signal stimulation. The control system was designed using Lyapunov functions and Linear Matrix Inequalities (LMIs) to obtain the control low gains.

The feedback signal, related to the knee joint angle, can be obtained by using an electrogoniometer. However, this signal may not be reliable due to the difficulty of adhering the device to the skin (Dejnabadi et al., 2005; Gaino, 2009). The use of accelerometers in strategic points is more stable and reliable (Dejnabadi et al., 2005; Gaino, 2009), and allows the control of nonlinear plants described by T-S fuzzy models, using state derivative feedback (Cardim et al., 2007a; Gaino, 2009). The objective of this work was the use of T-S fuzzy control techniques in simulation of rehabilitation engineering applications, in which the available control signals can be obtained by using accelerometers.

\section{Methods}

The lower limb was modeled as an open kinematic chain given by two rigid segments: the thigh and shank-foot complex, as shown in Figure 1 (Ferrarin and Pedotti, 2000). It was considered that the electrically stimulated quadriceps muscle exhibits a non-linear response (Crago et al., 1980). This nonlinear model relates the width of the applied pulse to the knee joint angle. The plant was described by a T-S fuzzy model.

The fuzzy regulator design was based on the Parallel Distributed Compensation (PDC) (Tanaka et al., 1998) and LMIs (Linear Matrix Inequalities) (Gahinet et al., 1995). This method enables the fuzzy combination of the feedback gain matrices, resulting in a fuzzy regulator that can stabilize the whole system (Teixeira and Żak, 1999). The regulator was designed for an operation at $30^{\circ}$, i.e., the leg goes from a resting state and stabilizes at $30^{\circ}$. In this work, two accelerometers were used to obtain the tangential accelerations $a_{t l}$ and $a_{t 2}$. Figure 2 shows how the accelerometers are attached to the leg (Franken et al., 1993). The acceleration of the knee joint $\ddot{\theta}_{v}$ is determined by the equation $\ddot{\theta}_{v}=\left(a_{t 1}-a_{t 2}\right) /\left(r_{1}-r_{2}\right)$.

The speed can be obtained by integrating the acceleration of the knee joint. The state derivative feedback was used in the controller design (Cardim et al., 2007a), described by a nonlinear T-S fuzzy model.

Setting the speed and displacement as state variables, the state derivative signals of the system are available. For the control design, first the displacement is estimated using Equation 17, obtained from the system Equation 1. This estimation is necessary for the implementation of the control input given in Equation 4.

\section{Results}

Using accelerometers, the system's feedback was performed using the estimation of the position $x_{p}$, correspondent to knee joint position, based on Equation 11. The operation point, used in the control design, was the following: $\theta_{v 0}=\pi / 6 \mathrm{rad}\left(30^{\circ}\right)$, $M_{a 0}=4.6068 \mathrm{Nm}$ and $P_{0}=M_{a 0} / G=1.0839 \cdot 10^{-4} \mathrm{~s}$. The controller gains, considering the conditions of Lemma 1, a decay rate $\beta=1.4$ and an input constraint $|u(t)| \leq \mu$, where $\mu=500 \cdot 10^{-6}$, are given in Equation 27.

The results of the controlled knee joint angle variation $\left(\theta_{v}\right)$ of a paraplegic patient, using the exact curve (continuous), represented by Equation 2 and the approximate curve (dashed) given in Figure 3, are illustrated in Figure 6. Note that the system converged to the desired point of balance, $0.52 \mathrm{rad}\left(30^{\circ}\right)$. Figure 7 shows other simulation results: angular position of the knee joint, angular velocity of the knee joint, torque produced by electrical stimulation to the quadriceps muscle and pulse width of the wave form.

Figure 8 shows the behavior of the system, without applying the control law, i.e., when the pulse width becomes void, at the $30^{\circ}$ position. The leg returns to the resting position by the action of gravity. The simulation results given in Figures 7 and 8 show that the controlled system satisfies the design specifications.

\section{Discussion}

From the accelerometers signals it is possible to reconstruct the velocities with good accuracy, but not the displacements. Considering this fact, a new control design method to vary the joint knee angle of paraplegic patients using FES, based on the T-S fuzzy models and using state-derivative feedback was proposed. The stability analysis of the controlled system used a Lyapunov function. The design was based on LMI that allows the specification of the stability, decay rate and input constraint. Simulations results show that this procedure presents good results.

\section{Conclusion}

This paper shows the feasibility of designing control systems for the position control of paraplegic legs using feedback signals produced by accelerometers. Accelerometers are very suitable for these applications due their small dimension, weight, and cost. As far as we know, this paper describes the first attempt to apply accelerometers, considering the nonlinear plant described by T-S fuzzy model and using state derivative feedback. Simulation results show that the proposed method is effective, in the position control of the leg of a paraplegic patient, for the knee joint angle between $0^{\circ}$ and $30^{\circ}$. 


\section{Introdução}

A Estimulação Elétrica Funcional tem sido utilizada na restauração de movimentos em pacientes paraplégicos, tetraplégicos e hemiplégicos. Existem vários estudos neste assunto, com diferentes modelos matemáticos de músculos e da articulação do joelho para o controle do movimento (Crago et al., 1980; Ferrarin e Pedotti, 2000; Gaino, 2009; Hill, 1938; Riener e Fuhr, 1998; Winter, 2005).

Em Gaino (2009) e Teixeira et al. (2006a,b) foram realizados, pela primeira vez, estudos e simulações da posição da perna de pacientes paraplégicos, utilizando modelos fuzzy Takagi-Sugeno (T-S) (Takagi e Sugeno, 1985; Tanaka et al., 1998; Teixeira e Żak, 1999). O uso do sistema em malha fechada possibilita a otimização da largura de pulso do neuroestimulador, diminuindo a fadiga do músculo (Crago et al., 1980; Riener e Fuhr, 1998).

O sinal de realimentação, relacionado ao ângulo da articulação do joelho, pode ser obtido utilizando-se eletrogoniômetro. No entanto, a utilização de acelerômetros simplifica as medições, devido à maior facilidade de fixação na pele do paciente. O uso de acelerômetros em pontos estratégicos é mais seguro e confiável (Dejnabadi et al., 2005; Gaino, 2009), e possibilita o controle de plantas não-lineares descritas por meio de modelos fuzzy T-S, usando a realimentação das derivadas das variáveis de estado (Cardim et al., 2007a; Gaino, 2009). Adicionalmente, os acelerômetros são adequados para essas aplicações, pois são relativamente pequenos, leves e baratos.

O objetivo deste trabalho é mostrar a vantagem do uso de acelerômetros, em vez de eletrogoniômetros, utilizando uma técnica de controle não-linear por meio dos modelos fuzzy T-S (Takagi e Sugeno, 1985). Nas simulações realizadas em MATLAB ${ }^{\circledR}$, os sinais disponíveis para o projeto de controle são representados pelas variáveis de estado da planta, como o deslocamento (obtido pelo eletrogoniômetro), a velocidade (obtida pela integração do sinal da aceleração, fornecido pelo acelerômetro), e o torque ativo produzido pela estimulação elétrica no quadríceps (obtido pela célula de carga, com strain gauge). O sinal da velocidade, obtido pela integração da aceleração, foi utilizado em Cardim et al. (2007a) e Gaino (2009) para o projeto de controle através da realimentação derivativa (Assunção et al., 2007; Cardim et al., 2007a,b; 2008; Faria et al., 2009a,b; Gaino, 2009).

Até o momento não foram encontrados na literatura trabalhos sobre realimentação derivativa no controle de plantas, usando modelos fuzzy T-S, com o propósito de controlar o movimento dos membros inferiores de pacientes paraplégicos.

\section{Métodos}

Neste trabalho, foi adotado o modelo matemático da perna proposto por Ferrarin e Pedotti (2000), que relaciona o torque do músculo com a largura de pulso do sinal de estimulação elétrica e expandido em espaço de estados (Gaino, 2009). Considerou-se que o músculo quadríceps eletricamente estimulado apresenta uma resposta não-linear (Crago et al., 1980). No projeto do controlador, foi usado um modelo fuzzy T-S da planta para simular a variação do ângulo da articulação do joelho, como mostrado em Gaino (2009). O projeto do regulador fuzzy, que considerou apenas a obtenção de informações por meio de sensores acelerométricos, foi elaborado através da Compensação Paralela Distribuída (CPD) (Tanaka et al., 1998), utilizando-se Desigualdades Matriciais Lineares (Linear Matrix Inequalities - LMIs) (Gahinet et al., 1995) para a obtenção dos ganhos do regulador. Este método faz a combinação fuzzy das matrizes de ganho de retroação, obtendo-se um regulador que pode estabilizar o sistema globalmente (Teixeira e Żak, 1999). O projeto do regulador foi realizado para o ponto de operação de $30^{\circ}$, isto é, a trajetória da perna sai do estado de repouso e estabiliza-se em $30^{\circ}$ (Gaino, 2009).

\section{Modelo da articulação do joelho}

Na modelagem proposta por Ferrarin e Pedotti (2000), os autores consideram o membro inferior como uma cadeia cinemática aberta composta de dois segmentos rígidos: a coxa e o complexo perna-pé, conforme mostrado na Figura 1. Nesta figura, a eletroestimulação funcional é aplicada ao músculo quadríceps, por meio de eletrodos fixados na pele da coxa do paciente. O objetivo é provocar a contração do músculo, visando posicionar o ângulo do joelho em um valor especificado.

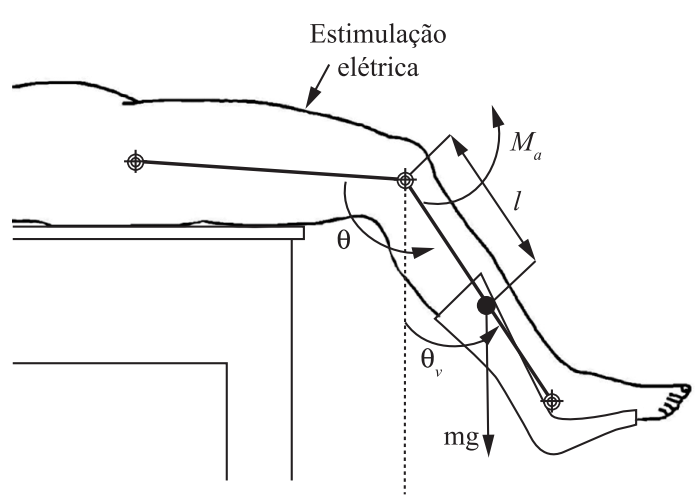

Figura 1. Sistema de controle e parâmetros $\theta, \theta_{v}$ e $M_{a}$. Figure 1. Control system and parameters $\theta, \theta_{v}$ and $M_{a}$. 
Na Figura 1, $\theta, \theta_{\mathrm{v}}$ e $M_{\mathrm{a}}$ são, respectivamente, o ângulo do joelho (ângulo entre a perna e a coxa no plano sagital), o ângulo da perna (ângulo entre a perna e o eixo vertical no plano sagital) e o torque ativo produzido pela estimulação elétrica no quadríceps.

Em Gaino (2009) e Teixeira et al. (2006a,b), demonstrou-se que a equação de estados é não-linear, e que representa o movimento da articulação do joelho em decorrência de estímulo elétrico aplicado no quadríceps, sendo dada por:

$\left[\begin{array}{l}\dot{x}_{1} \\ \dot{x}_{2} \\ \dot{x}_{3}\end{array}\right]=\left[\begin{array}{ccc}0 & 1 & 0 \\ \tilde{f}_{21}\left(x_{1}\right) & \frac{-B}{J} & \frac{1}{J} \\ 0 & 0 & \frac{-1}{\tau}\end{array}\right]\left[\begin{array}{l}x_{1} \\ x_{2} \\ x_{3}\end{array}\right]+\left[\begin{array}{c}0 \\ 0 \\ \frac{G}{\tau}\end{array}\right] P_{N}$

sendo $x_{1}=\Delta \theta_{\mathrm{v}}=\theta_{\mathrm{v}}-\theta_{\mathrm{v} 0}, x_{2}=\dot{x}_{1}, x_{3}=\Delta M_{a}=M_{a}-M_{a 0}$, $P_{N}=P-\frac{M_{a 0}}{G}$ e o ponto de operação do sistema dado $\operatorname{por}\left(\theta_{v}, \dot{\theta}_{v}, M_{a}\right)-\left(\theta_{v 0}, 0, M_{a 0}\right)$. A função $\widetilde{f}_{21}\left(x_{1}\right)$ é uma não-linearidade do sistema, que pode ser descrita por:

$$
\begin{aligned}
& \tilde{f}_{21}\left(x_{1}\right)=\frac{1}{J x_{1}}\left[\begin{array}{l}
-m g l \operatorname{sen}\left(x_{1}+\theta_{v 0}\right)-\lambda e^{-E\left(x_{1}+\theta_{v 0}+\frac{\pi}{2}\right)} \\
\left(x_{1}+\theta_{v 0}+\frac{\pi}{2}-\omega\right)+M_{a 0}
\end{array}\right], \\
& M_{a 0}=m g l \operatorname{sen}\left(\theta_{v 0}\right)+\lambda e^{-E\left(\theta_{v 0}+\frac{\pi}{2}\right)}\left(\theta_{v 0}+\frac{\pi}{2}-\omega\right) \\
& P_{N}=P-\frac{M_{a 0}}{G}
\end{aligned}
$$

sendo que $G$ e $\tau$ são constantes da equação diferencial que relacionam o torque ativo com a estimulação elétrica, e:

- J é o momento inercial do complexo perna-pé;

- $\theta_{v}$ é o ângulo da perna (ângulo entre a perna e o eixo vertical no plano sagital);

- $m$ é a massa do complexo perna-pé;

- l é a distância entre o joelho e o centro de massa do complexo perna-pé;

- $B$ é o coeficiente de atrito viscoso;

- $P$ é a largura do pulso;

- $P_{\mathrm{N}}$ é a entrada do sistema.

Em Ferrarin e Pedotti (2000), foi feita a identificação dos parâmetros $G$ e $\tau$ da função de transferência que relaciona a largura de pulso e o torque produzido. Este modelo refere-se à aplicação de estímulos no músculo quadríceps, pretendendo-se posicionar o ângulo da articulação do joelho em um valor desejado em malha fechada, para exercitá-lo. No entanto, para outras aplicações, como controle de postura ereta do paciente, este modelo do Ferrarin e Pedotti (2000) é limitado e deve ser adaptado. Um modelo mais complexo pode ser considerado, como em Naves (2006), que usa controladores PID em malha fechada para controle da postura de pacientes.

$\mathrm{Na}$ Equação 3 é definida a nova entrada do sistema $P_{\mathrm{N}}$, com o objetivo de estudar a estabilidade do sistema controlado com uma função de Lyapunov. A entrada original do sistema era a largura de pulso $P$.

Em Ferrarin e Pedotti (2000) são apresentadas as medidas antropométricas de um paciente paraplégico:

- $\tau=0,951 \mathrm{~s} ; B=0,27 \mathrm{~N} \cdot \mathrm{m} \cdot \mathrm{s} / \mathrm{rad} ; \omega=2,918 \mathrm{rad} ;$ $\lambda=41,208 \mathrm{~N} \cdot \mathrm{m} / \mathrm{rad} ; E=2,024 \mathrm{rad}^{-1}$; $J=0,362 \mathrm{~kg} \cdot \mathrm{m}^{2} ; m=4,37 \mathrm{~kg} ; l=23,8 \mathrm{~cm}$.

Este modelo de Ferrarin e Pedotti (2000) fornece, pela identificação da função de transferência, a relação torque produzido pelo músculo por largura de pulso, que é uma simplificação importante para estudo na área de controle. Em Ferrarin e Pedotti (2000), estudaram-se padrões de estimulação com amplitude e frequência fixas e largura de pulso variável, e assim mostrou-se que um modelo com um pólo pode representar bem a complexidade fisiológica para gerar o torque. São utilizados neste modelo eletrodos de superfície, fixados no músculo quadríceps. Informações sobre o formato e a distância entre eletrodos podem ser encontradas em Crago et al. (1980), e Ferrarin e Pedotti (2000).

\section{Definição do problema}

Considerando o modelo fuzzy T-S como descrito na Equação 4 (Takagi e Sugeno, 1985):

$$
\begin{gathered}
\dot{x}(t)=\sum_{i=1}^{r} \alpha_{i}(x(t))\left(A_{i} x(t)+B_{i} u(t)\right)= \\
A(\alpha(x(t))) x(t)+B(\alpha(x(t))) u(t), \\
u(t)=-\sum_{i=1}^{r} \alpha_{i}(x(t)) F_{i} x(t)=-F(\alpha(x(t))) x(t), \\
\sum_{i=1}^{r} \alpha_{i}(x(t))=1, \quad \alpha_{i}(x(t)) \geq 0, \quad i=1,2, \ldots, r
\end{gathered}
$$

sendo $r$ o número de modelos locais $\left(A_{\mathrm{i}}, B_{\mathrm{i}}, i=1,2, \ldots, r\right)$, $a_{\mathrm{i}}(x(t))$ as funções de pertinência do sistema fuzzy, $F_{i}$ os ganhos de retroação do vetor de estado obtidos por meio de LMIs (Gahinet et al., 1995) e $u(t)$ o sinal de controle, obtido por meio de CPD. Supondo que apenas a derivada do vetor de estado $(\dot{x})$ esteja disponível, verifica-se na Equação 4 a necessidade de medir o estado $x$ para o projeto das funções de pertinências e do ganho $F(\alpha(x(t)))$ para o controlador fuzzy. O objetivo do projeto do regulador fuzzy é determinar os ganhos de realimentação locais $F_{i}$ nas partes consequentes. No modelo do paciente paraplégico, existe apenas uma não-linearidade e, portanto, de acordo com a modelagem exata de Taniguchi et al. (2001), haverá dois modelos locais. Os valores mínimos e máximos de $\widetilde{f}_{21}\left(x_{1}\right)$, mostrados em Gaino (2009) e Teixeira et al. (2006b), para $x_{1}$ variando no intervalo de $-30^{\circ}$ até $30^{\circ}$ são: 
- $a_{221}=\max \left\{\tilde{f}_{21}\left(x_{1}(t)\right)\right\}=-22,20124$;

- $a_{212}=\min \left\{\tilde{f}_{21}\left(x_{1}(t)\right)\right\}=-36,50663$.

A partir dos valores máximo e mínimo de $\widetilde{f}_{21}\left(x_{1}\right)$, obtêm-se as funções de pertinência e os modelos locais (Taniguchi et al., 2001):

$$
\begin{aligned}
& \tilde{f}_{21}\left(x_{1}\right)=\alpha_{1}\left(x_{1}(t)\right) \cdot a_{211}+\alpha_{2}\left(x_{1}(t)\right) \cdot a_{212} \\
& \alpha_{1}\left(x_{1}(t)\right)=\frac{\tilde{f}_{21}\left(x_{1}\right)-a_{212}}{a_{211}-a_{212}}, \alpha_{2}\left(x_{1}(t)\right)=\frac{\tilde{f}_{21}\left(x_{1}\right)-a_{211}}{a_{212}-a_{211}}
\end{aligned}
$$

$A_{1}=\left[\begin{array}{ccc}0 & 1 & 0 \\ a_{211} & \frac{-B}{J} & \frac{1}{J} \\ 0 & 0 & \frac{-1}{\tau}\end{array}\right], A_{2}=\left[\begin{array}{ccc}0 & 1 & 0 \\ a_{212} & \frac{-B}{J} & \frac{1}{J} \\ 0 & 0 & \frac{-1}{\tau}\end{array}\right], B_{1}=B_{2}=\left[\begin{array}{c}0 \\ 0 \\ \frac{G}{\tau}\end{array}\right]$

Analisando as Equações 6, verifica-se que as funções de pertinência dependem apenas da variável de estado $x_{1}$, e que a não-linearidade também depende somente de $x_{1}$. Compondo os modelos locais, o sistema (1) é equivalente ao sistema proposto em Taniguchi et al. (2001):

$$
\begin{gathered}
\dot{x}(t)=\sum_{i=1}^{2} \alpha_{i}\left(x_{1}(t)\right) A_{i} x(t)+\sum_{i=1}^{2} \alpha_{i}\left(x_{1}(t)\right) B_{i} u(t)= \\
A(\alpha(x(t))) x(t)+B(\alpha(x(t))) u(t)
\end{gathered}
$$

sendo $u(t)=P_{N}(t)$.

Supondo que somente acelerômetros sejam utilizados no sistema, a aceleração $\dot{x}_{2}$ está disponível. É possível, a partir de $\dot{x}_{2}$, estimar com boa exatidão a velocidade $x_{2}$, mas não a posição $x_{1}$ (Abdelaziz e Valášek, 2004). Neste caso, verifica-se por meio das Equações 6 que a variável de estado $x_{1}$ é necessária para o cálculo das funções de pertinência $\alpha_{i}(x(\mathrm{t}))$, $i=1,2$ e o sistema de controle por realimentação, que utiliza essas funções, não pode ser diretamente implementado. Uma forma de resolver esse problema é proposta neste trabalho, usando o método de inversão descrito na próxima seção, para estimar a variável $x_{1}$.

\section{Obtenção da aceleração e da velocidade com acelerômetros}

Os resultados publicados em Gaino (2009) e Teixeira et al. $(2006 \mathrm{a}, \mathrm{b})$ consideram a realimentação do sistema com eletrogoniômetros, nos quais uma de suas extremidades é presa à coxa e a outra à perna. Devido à dificuldade de se alinhar a haste do eletrogoniômetro com o eixo da coxa e a outra haste com o eixo da perna, e ainda o efeito do deslizamento sobre a pele, as medidas não são confiáveis (Winter, 2005). Para resolver esse problema, acelerômetros são fixados em pontos estratégicos, conforme descrito em Dejnabadi et al. (2005), que obtiveram resultados mais confiáveis, mostrando que acelerômetros e giroscópios oferecem soluções mais práticas e menos onerosas quando comparados com eletrogoniômetros. No presente trabalho utilizam-se dois acelerômetros para medir as acelerações tangenciais $a_{\mathrm{t} 1} \mathrm{e} a_{\mathrm{t} 2}$. A Figura 2 ilustra a posição dos acelerômetros fixados na perna do paciente, segundo as especificações de Franken et al. (1993).

A aceleração da articulação do joelho $\ddot{\theta}_{v}$ é determinada pela equação:

$\ddot{\theta}_{v}=\frac{\left(a_{t 1}-a_{t 2}\right)}{\left(r_{1}-r_{2}\right)}$

A velocidade pode ser obtida integrando-se esta equação. Em Dejnabadi et al. (2005) e Franken et al. (1993) são sugeridas algumas técnicas para se obter a velocidade angular.

\section{Estimação da posição da perna do paciente paraplégico}

Como somente acelerômetros são usados, só há acesso à variável $\dot{x}_{2}=\ddot{x}_{1}$. Utilizando-se o sinal $\dot{x}_{2}=\ddot{x}_{1}$, a variável $x_{2}=\dot{x}_{1}$ é estimada, mas a estimação de $x_{1}$ não é precisa (Abdelaziz e Valášek, 2004). No projeto do controlador apresentado em Gaino (2009) e Teixeira et al. (2006a,b), a sua implementação só é possível com o acesso a $x_{1}$, necessário para o cálculo das funções de pertinências $\alpha_{i}\left(x_{1}(t)\right), i=1,2$. Observando as Equações 5, 6 e 8, nota-se que elas são expressas em função de $x_{1}$. O objetivo é estimar $x_{1}$ para a estimação de $\alpha_{i}\left(x_{1}(t)\right), i=1,2$. Considerando a Equação 1, a estimação de $x_{1}$ pode ser analisada por meio da seguinte equação:

$\dot{x}_{2}=\tilde{f}_{21}\left(x_{1}\right) x_{1}-\frac{B}{J} \dot{x}_{1}+\frac{1}{J} x_{3}$

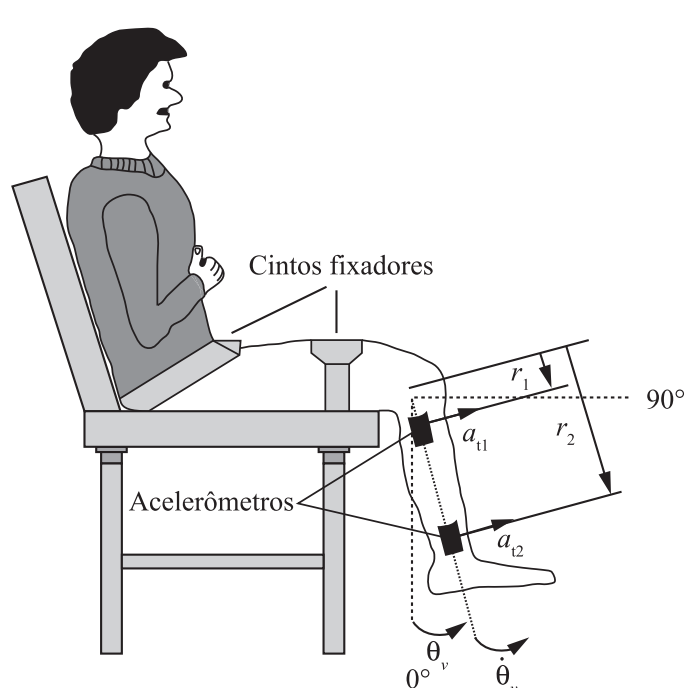

Figura 2. Posição dos acelerômetros para medida da aceleração tangencial.

Figure 2. Position of the accelerometers for measuring the tangential acceleration. 
A variável $x_{3}$ refere-se ao torque produzido pela estimulação elétrica. Essa variável está disponível, pois pode ser medida por meio de sensores de força. A função não-linear na Equação 2, para $x_{1}\left(x_{1}=\theta_{v}-\theta_{v 0}\right)$ variando no intervalo $0^{\circ}<\theta_{v}<60^{\circ} \operatorname{com} \theta_{v 0}=30^{\circ}$, pode ser aproximada por uma reta usando a ferramenta computacional "basic fitting tool of Matlab". Com o uso de uma interação gráfica, pode-se escolher tipos de interpoladores para aproximação de curvas a partir dos pares $(x, y)$ gerados no gráfico. Uma aproximação de primeira ordem de $\widetilde{f}_{21}\left(x_{1}\right)$, com $x_{1} \in\left[-30^{\circ}, 30^{\circ}\right]$, foi obtida graficamente e pode ser escrita como: $\widetilde{f}_{21}\left(x_{1}\right) \approx a x_{1}+b$. Então, tem-se $\tilde{f}_{21}\left(x_{1}\right) x_{1} \approx\left(a x_{1}+b\right) x_{1}$. Tomando este fato como base, utilizou-se a equação $\widetilde{f}_{21}\left(x_{1}\right) x_{1} \approx\left(a x_{1 e}+b\right) x_{1 e}$ para a estimação de $x_{1}$, representada por $x_{1 e}$. Desta forma, a Equação 10 pode ser reescrita como:

$\dot{x}_{2}=\left(a x_{1 e}+b\right) x_{1 e}-\frac{B}{J} \dot{x}_{1}+\frac{1}{J} x_{3}, \tilde{f}_{21}\left(x_{1}\right) x_{1}=\left(a x_{1 e}+b\right) x_{1 e}$

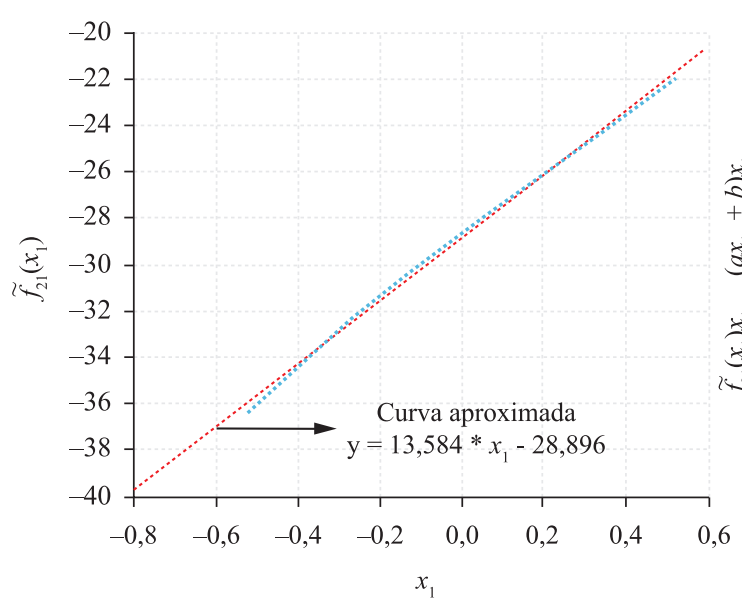

(a)

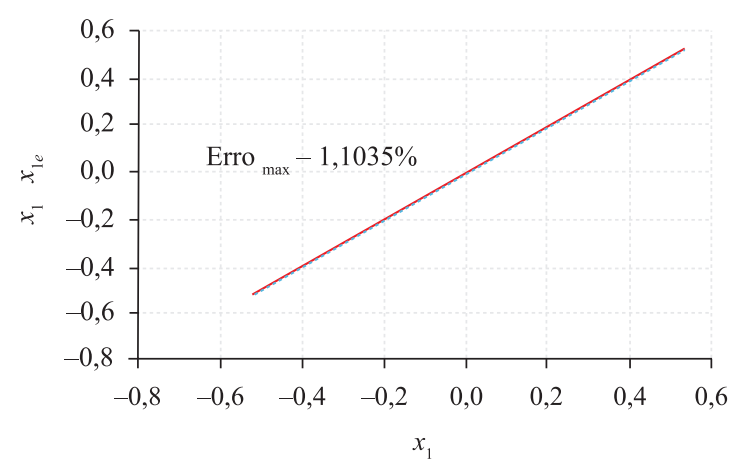

sendo que $x_{1 e}$ é o valor estimado de $x_{1}$.

Na Figura 3a, o resultado da curva aproximada pelo "basic fitting tool of Matlab" fornece os coeficientes $a=13,584 \mathrm{e} b=-28,896$. A Figura $3 \mathrm{~b}$ mostra a curva $\widetilde{f}_{21}\left(x_{1}\right) x_{1}$ e a forma aproximada $\left(a x_{1}+b\right) x_{1}$. Verifica-se que a aproximação foi boa, com um erro relativo $\left(\frac{100\left|\tilde{f}_{21}\left(x_{1}\right) x_{1}-\left(a x_{1}+b\right) x_{1}\right|}{\left|\tilde{f}_{21}\left(x_{1}\right) x_{1}\right|}\right)$ máximo de $1,39 \%$. Da Equação 11 obtém-se:

$a x_{1 e}^{2}+b x_{1 e}-\frac{B}{J} \dot{x}_{1}-\dot{x}_{2}+\frac{1}{J} x_{3}=0$

Definindo

$$
c(t)=-\frac{B}{J} \dot{x}_{1}-\dot{x}_{2}+\frac{1}{J} x_{3}
$$

então,

$$
a x_{1 e}^{2}+b x_{1 e}+c(t)=0
$$

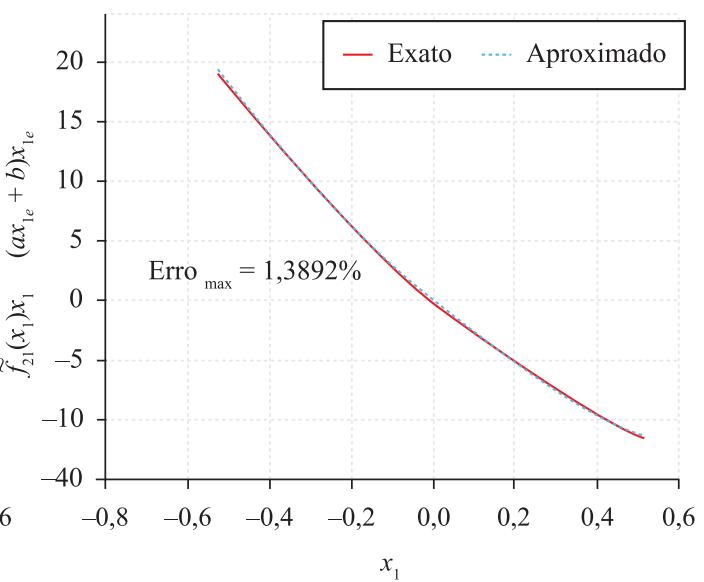

(b)

(c)

Figura 3. a) Curvas exata e aproximada da função não-linear $\tilde{f}_{21}\left(x_{1}\right)$; b) Curvas exata e aproximada de $\tilde{f}_{21}\left(x_{1}\right) x_{1}$ com erro máximo de aproximação; e c) Curvas exata e aproximada de $x_{1}$ com erro máximo de aproximação.

Figure 3. a) Exact and approximate curves of the nonlinear function $\widetilde{f}_{21}\left(x_{1}\right)$; b) Exact and approximate curves of $\widetilde{f}_{21}\left(x_{1}\right) x_{1}$ with maximum approach error; and c) Exact and approximate curves of $x_{1}$ with maximum approach error. 
Considerando as Equações 11 e 13, $c(t)=-x_{1} \widetilde{f}_{21}\left(x_{1}\right)$, e, considerando a Equação 14, $x_{1 e}$ é dada por:

$$
x_{1 e}=\frac{-b-\sqrt{b^{2}-4 a c(t)}}{2 a}=\frac{-b-\sqrt{b^{2}+4 a x_{1} \tilde{f}_{21}\left(x_{1}\right)}}{2 a}
$$

A Figura $3 \mathrm{c}$ mostra que o erro relativo máximo da estimação de $x_{1}$, no intervalo $x_{1} \in\left[\frac{-\pi}{6}, \frac{\pi}{6}\right]$, foi de $1,10 \%$. Note que, da Equação 14, supondo que $a x_{1 e}^{2}+b x_{1 e} \neq 0$, então a equação característica é dada por:

$1+\frac{c(t)}{a x_{1 e}^{2}+b x_{1 e}}=0$

Como $c(t)$ é um parâmetro livre, as raízes características do sistema devem satisfazer a Equação 16. Na Figura 4 estão mostradas as raízes de $x_{1 e}$ em função de $c(t)$ (Dorf e Bishop, 2001). Então, da Equação 15, para a solução do problema $\left(x_{1 e} \in \Re, x_{1} \in\left[\frac{-\pi}{6}, \frac{\pi}{6}\right]\right)$, o lugar das raízes da equação característica 16 deve ser real no intervalo $[-\pi / 6, \pi / 6]$, quando variar $c(t)$. Pela análise do lugar das raízes, a posição dos pólos em malha fechada, quando o parâmetro varia para $c(t)>0$, é mostrada na Figura 4. Na Figura 5, é mostrada a localização dos pólos em malha fechada com a variação para $c(t)<0$.

Analisando-se os resultados das Figuras 4 e 5, o lugar das raízes existe para o intervalo $-\pi / 6 \leq x_{1 e} \leq \pi / 6$, quando $-18,00 \leq c(t) \leq 11,05$. Consequentemente, verificando a Equação 15 , a solução analítica de $x_{1 e}$ existe dentro do intervalo $[-\pi / 6, \pi / 6]$ e encontra-se como solução numérica:

$$
\begin{aligned}
x_{1 e}= & \sqrt{1,1313}-\sqrt{1,1313-0,0736 c(t)}= \\
& \sqrt{1,1313}-\sqrt{1,1313+0,0736 x_{1} \tilde{f}_{21}\left(x_{1}\right)}
\end{aligned}
$$

Desse modo, $x_{1}$ pode ser estimado utilizando os sinais $\dot{x}_{1}, \dot{x}_{2}$ e $x_{3}$. Todas essas variáveis estão disponíveis por meio de sensores.

Note que a estimação de $x_{1}$ utilizando a Equação 11, considerando a Equação 13 e dada na 17, independe explicitamente da entrada $P_{N}$ do sistema (Equação 1) ou seja, não existe looping algébrico nesta estimação. Este fato pode ser facilmente confirmado, lembrando-se que nesta estimação foram utilizadas as Equações 10 e 11, provenientes da equação da planta 1 e observandose que $\dot{x}_{1}=x_{2}$. Desta forma, o uso de $x_{1}$ estimado na obtenção da lei de controle $P_{N}$ não afeta a sua estimação. Este fato também ocorre no problema clássico de realimentação de estado com observador, conhecido como princípio da separação, no qual os projetos da estimação e controle podem ser tratados independentemente, quando não existem incertezas na planta (Ogata, 2003). Uma análise teórica e simulações

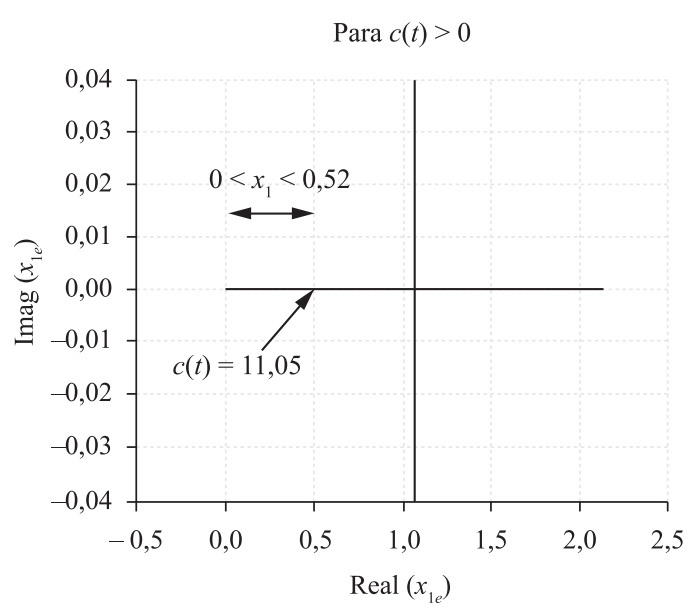

Figura 4. Lugar das raízes $\operatorname{com} c(t)>0$.

Figure 4. Root locus with $c(t)>0$.

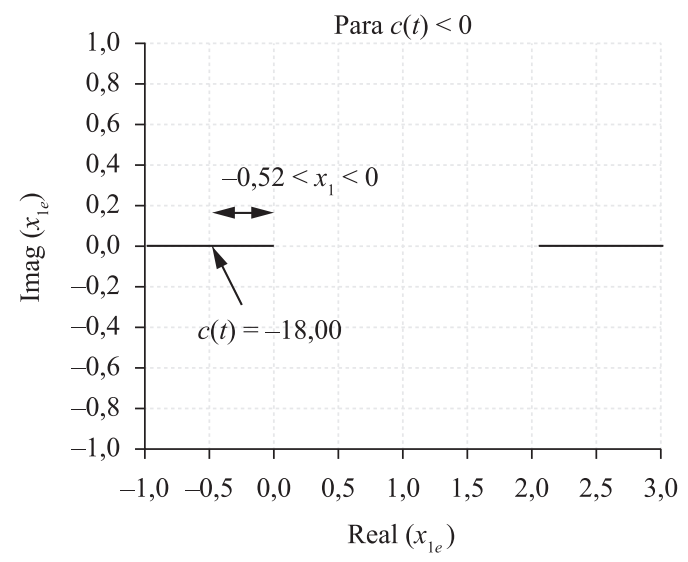

Figura 5. Lugar das raízes $\operatorname{com} c(t)<0$.

Figure 5. Root locus with $c(t)<0$.

digitais do sistema de controle proposto, apresentadas na seção Resultados, mostram que este pequeno erro não afeta o desempenho do sistema.

\section{Controle da posição da perna com modelos fuzzy $T-S$}

O conceito de Compensação Paralela Distribuída (CPD) (Tanaka et al., 1998) foi usado para projetar reguladores que estabilizam sistemas não-lineares descritos por meio de modelos fuzzy T-S, como descrito na Equação 4. A idéia é projetar um regulador para cada regra do modelo fuzzy. O modelo global é uma combinação fuzzy dos reguladores projetados. Este modelo global foi apresentado na Equação 4, podendo-se obter mais detalhes em Gaino (2009) e Teixeira et al. (2006a,b). O regulador é determinado por meio da resolução de LMIs, segundo critérios apresentados em Tanaka et al. (1998) e desenvolvidos para o caso do controle do movimento de paraplégicos em Teixeira et al. (2006a,b). 
Lema 1: O controle CPD (Tanaka et al., 1998), que considera simultaneamente a estabilidade e a taxa de decaimento $\beta$, sendo $\beta>0$ (quanto maior for $\beta$, mais rápido será o transitório) para o sistema controlado, pode ser especificado se existirem uma matriz simétrica positiva definida $X\left(X>0, X=X^{T}\right)$, e uma matriz $M_{i}, i=1,2, \ldots, r$ tais que,

$$
X A_{i}^{T}-M_{i}^{T} B_{i}^{T}+A_{i} X-B_{i} M_{i}+2 \beta X<0
$$

$\mathrm{e}$

$$
\begin{aligned}
X A_{i}^{T}-M_{j}^{T} B_{i}^{T}+X A_{j}^{T}-M_{i}^{T} B_{j}^{T}+A_{i} X- \\
B_{i} M_{j}+A_{j} X-B_{j} M_{i}+4 \beta X<0 \\
i, j=1,2, \ldots, r, \quad i<j
\end{aligned}
$$

sendo que os ganhos do controlador são dados por $F_{i}=M_{i} X^{-1}, i=1,2, \ldots, r$. Considerando as condições iniciais conhecidas, então a restrição $|u(t)| \leq \mu$ é imposta para $t \geq 0$, para restrição do sinal de entrada do controlador, considerando as Equações 18 a 20, sendo $I$ uma matriz identidade (Boyd et al., 1994):

$\left[\begin{array}{cc}1 & x(0)^{T} \\ x(0) & X\end{array}\right] \geq 0$ e $\left[\begin{array}{cc}X & M_{i}^{T} \\ M_{i} & \mu^{2} I\end{array}\right] \geq 0$

Os modelos matemáticos têm suas aproximações e limitações. Por exemplo, a identificação linear do torque por largura de pulso é uma simplificação das propriedades função de ativação das fibras, relação força-comprimento e força-velocidade. A literatura mostra a dificuldade de obtenção destes modelos mais complexos. A análise matemática apresentada em Ferrarin e Pedotti (2000), quando implementada em malha fechada, deve ter o resultado próximo do simulado. Em Crago (1980) é apresentada uma técnica linear de controle com bons resultados, sendo a técnica linear uma particularização de técnicas não-lineares. A próxima seção mostra que o método proposto neste trabalho, utilizando os modelos fuzzy T-S, apresenta uma solução viável para implementação.

\section{Demonstração da estabilidade do sistema de controle proposto}

O sistema de controle proposto utiliza a estimação da variável de estado $x_{1}$, dada por $x_{1 e}$ em 15. Assim, é possível definir o vetor de estado estimado $x_{e}^{T}=\left[\begin{array}{lll}x_{1 e} & x_{2} & x_{3}\end{array}\right]$, sendo que o vetor de estado da planta 5-8 é $x^{T}=\left[\begin{array}{lll}x_{1} & x_{2} & x_{3}\end{array}\right]$. A lei de controle, tendo como base 4, pode agora ser escrita como:

$$
u(t)=-\sum_{i=1}^{2} \alpha_{i}\left(x_{e}(t)\right) F_{i} x_{e}(t)=-F\left(\alpha\left(x_{e}(t)\right)\right) x_{e}(t)
$$

sendo que $F_{1}$ e $F_{2}$ são projetadas utilizando-se o Lema 1 (que despreza o erro de estimação). Desta forma, note que o sistema controlado 8 e 21 é dado por:

$$
\begin{gathered}
\dot{x}=A(\alpha(x)) x+B(\alpha(x)) u=A(\alpha(x)) x+ \\
B(\alpha(x))\left(-F\left(\alpha\left(x_{e}\right)\right) x_{e}\right)=\left(\begin{array}{l}
A(\alpha(x)) x \\
-B F(\alpha(x)) x
\end{array}\right)+ \\
B\left(\begin{array}{l}
F(\alpha(x)) x \\
-F\left(\alpha\left(x_{e}\right)\right)
\end{array}\right) x+B F\left(\alpha\left(x_{e}\right)\right)\left(x-x_{e}\right)
\end{gathered}
$$

Para simplificar o equacionamento, utilizaremos a notação $x(t)=x$ e $x_{e}(t)=x_{e}$.

Considerando ainda, a candidata a função de Lyapunov $V(x)=x^{T} P x$, sendo que $P$ foi obtida utilizando-se as condições do Lema 1. Então, de 22:

$$
\begin{aligned}
\dot{V}(x) & =x^{T} P \dot{x}+\dot{x}^{T} P x=x^{T}\left[\begin{array}{c}
P(A(\alpha(x))-B F(\alpha(x)))+ \\
(A(\alpha(x))-B F(\alpha(x)))^{T} P
\end{array}\right] x \\
& +2 x^{T} P B\left\{\begin{array}{l}
\left(F(\alpha(x))-F\left(\alpha\left(x_{e}\right)\right)\right) x+ \\
F\left(\alpha\left(x_{e}\right)\right)\left[\begin{array}{c}
x_{1}-x_{1 e} \\
0 \\
0
\end{array}\right]
\end{array}\right\}
\end{aligned}
$$

Mas, as condições do Lema 1 implicam que:

$$
P\left(\begin{array}{l}
A(\alpha(x))- \\
B F(\alpha(x))
\end{array}\right)+\left(\begin{array}{l}
A(\alpha(x))- \\
B F(\alpha(x))
\end{array}\right)^{T} P \leq-2 \beta P
$$

sendo que no caso $\beta=1,4$. Adicionalmente, note que:

$$
\begin{gathered}
F\left(\alpha\left(x_{e}\right)\right)\left[\begin{array}{c}
x_{1}-x_{1 e} \\
0 \\
0
\end{array}\right]=F\left(\alpha\left(x_{e}\right)\right)\left[\begin{array}{l}
1 \\
0 \\
0
\end{array}\right]\left(1-\frac{x_{1 e}}{x_{1}}\right) x_{1}= \\
F\left(\alpha\left(x_{e}\right)\right)\left[\begin{array}{l}
1 \\
0 \\
0
\end{array}\right]\left(1-\frac{x_{1 e}}{x_{1}}\right)\left[\begin{array}{lll}
1 & 0 & 0
\end{array}\right] x= \\
\left(1-\frac{x_{1 e}}{x_{1}}\right) F\left(\alpha\left(x_{e}\right)\right)\left[\begin{array}{lll}
1 & 0 & 0 \\
0 & 0 & 0 \\
0 & 0 & 0
\end{array}\right] x
\end{gathered}
$$

Substituindo-se 24 e 25 em 23, note que:

$$
\begin{aligned}
& \dot{V}(x) \leq 2 x^{T} P\left\{B\left[\begin{array}{l}
\left(F(\alpha(x))-F\left(\alpha\left(x_{e}\right)\right)\right)+ \\
\left(\left(1-\frac{x_{1 e}}{x_{1}}\right) F\left(\alpha\left(x_{e}\right)\right)\left[\begin{array}{lll}
1 & 0 & 0 \\
0 & 0 & 0 \\
0 & 0 & 0
\end{array}\right]\right.
\end{array}\right]-\beta I\right\} x \\
& =2 x^{T} Z\left(x_{1}\right) x
\end{aligned}
$$

Utilizando a equação de $x_{1 e}$ dada em 15 , para $x_{1} \in\left[\frac{-\pi}{6}, \frac{\pi}{6}\right]$, observou-se que o máximo autovalor de $Z\left(x_{1}\right)$ foi $-1,3383$. Desta forma, de 26 conclui-se que $\dot{V}(x)<0$ para $\mathrm{x} \neq 0, V(0)=0 \mathrm{e}$, assim, o ponto de equilíbrio $x=0$ do sistema controlado 22 é assintoticamente estável. 


\section{Resultados}

Para a simulação do sistema, os parâmetros considerados foram descritos na seção do Modelo da Articulação do Joelho, e o projeto dos reguladores, descrito no Lema 1, garante a estabilidade do sistema

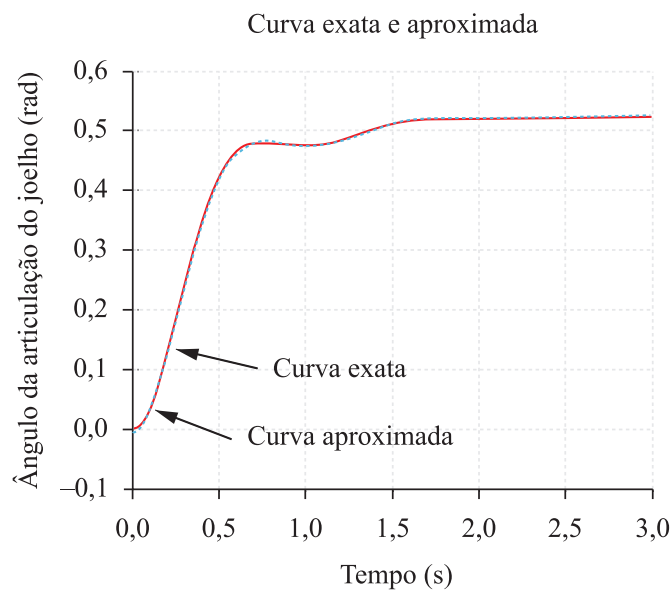

Figura 6. Variação do ângulo $\theta_{v}$ utilizando as funções $\widetilde{f}_{21}\left(x_{1}\right)$ exata e aproximada.

Figure 6. Variation of the angle $\theta_{v}$ using the exact and approximate functions $f_{21}\left(x_{1}\right)$.

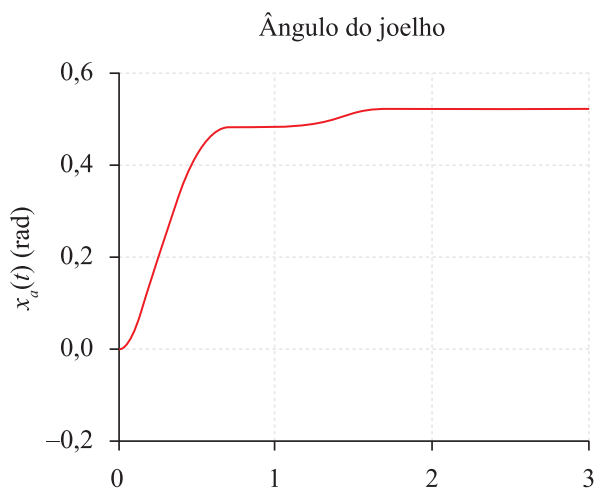

Torque ativo produzido pelo estímulo

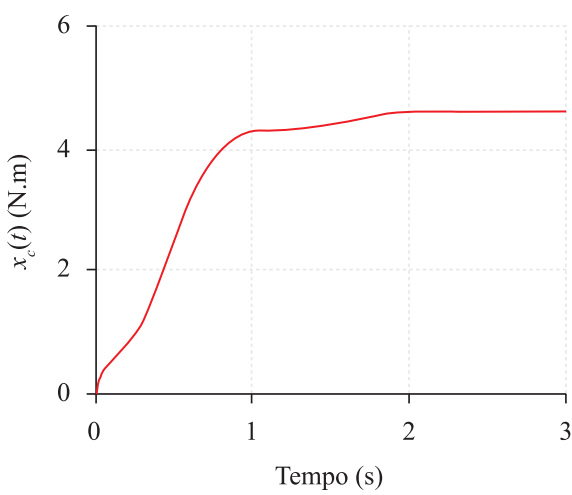

utilizando funções de Lyapunov $V(x)=x^{T} P x$, sendo $P=X^{-1}$, quando o vetor $x$ é disponível.

Nos gráficos das Figuras 6, 7 e 8 são apresentados os resultados da simulação, que foram obtidos a partir da realimentação usando acelerômetros, pelo método proposto neste trabalho.

Considerando as condições do Lema 1, os ganhos do controlador para $\beta=1,4$ e $\mu=50010^{6}$ são:

$F_{1}=10^{-3}\left[\begin{array}{lll}-0,6197 & 0,1286 & 0,1174\end{array}\right]$,

$F_{2}=10^{-3}\left[\begin{array}{lll}-0,8619 & 0,1347 & 0,1175\end{array}\right]$

A realimentação do sistema, utilizando acelerômetros, foi realizada com uso da estimação da posição $x_{1}$ por meio da Equação 17. As simulações da variação do ângulo da articulação do joelho $\theta_{v}$ de um paciente paraplégico, utilizando as curvas exata (contínua) e aproximada (tracejada), ilustradas na Figura 3, considerando as condições iniciais $x_{0}=\left[-\theta_{v 0} 0-\mathrm{M}_{a 0}\right]^{T}$, são ilustradas na Figura 6.

O uso da aproximação linear, para a função nãolinear na Equação 2, justifica-se pela facilidade de implementação computacional. Na Figura 6 observa-se que as respostas do sistema controlado com a função $\widetilde{f}_{21}\left(x_{1}\right)$ exata e aproximada, são praticamente idênticas,
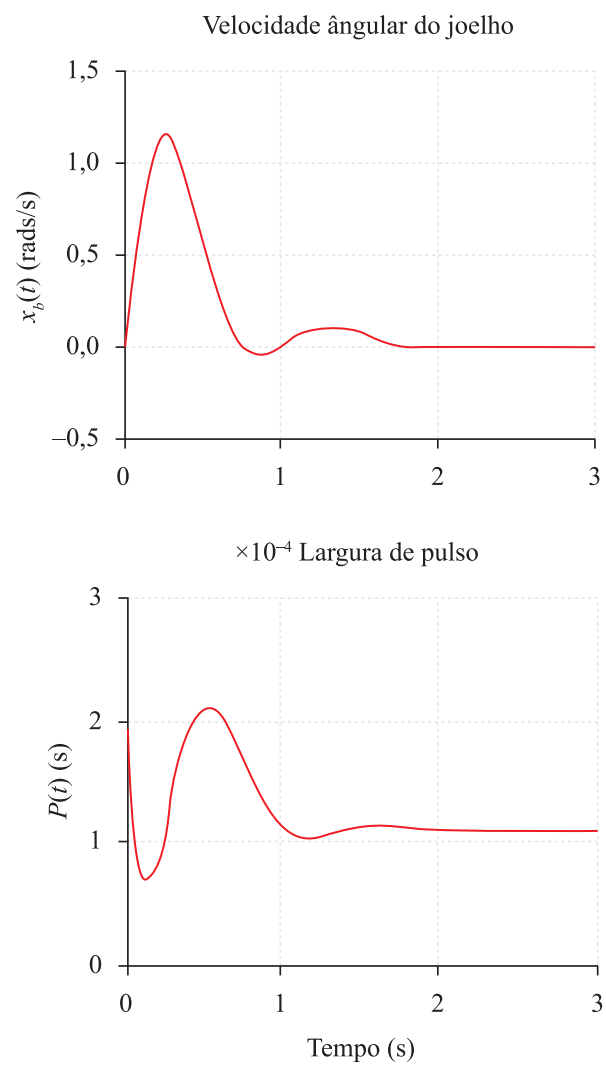

Figura 7. Resultado das simulações dos estados da planta com condições iniciais $x_{0}=\left[-\theta_{v 0}, 0-M_{\alpha 0}\right]^{T}$.

Figure 7. Simulation results of the states of the plant with the initial condition $x_{0}=\left[-\theta_{v 0}, 0-M_{a 0}\right]^{T}$. 
Ângulo do joelho

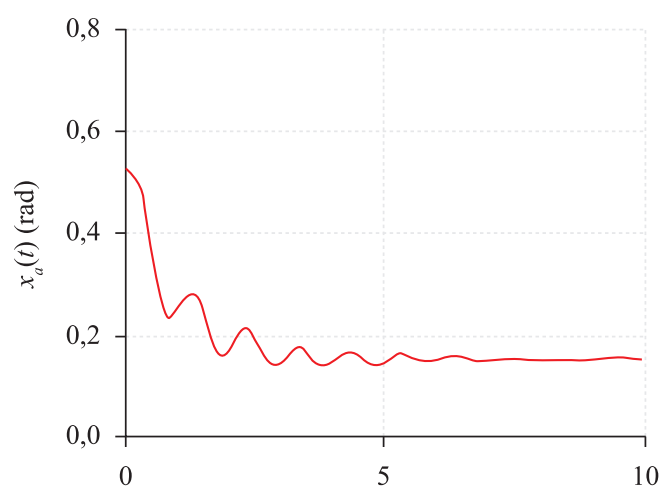

Torque ativo produzido pelo estímulo

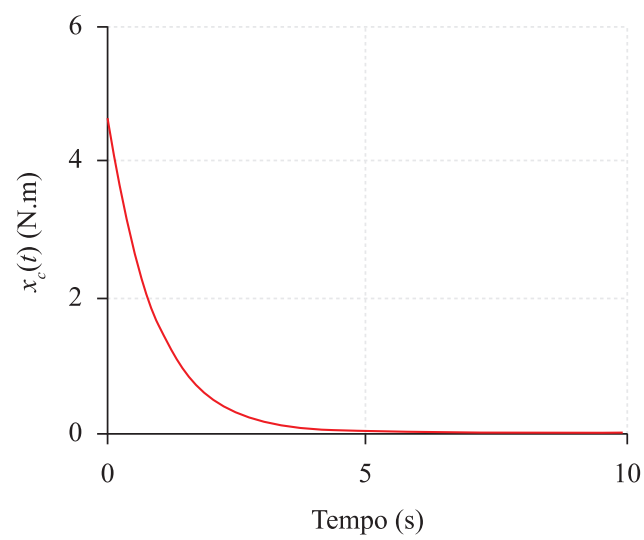

Velocidade ângular do joelho

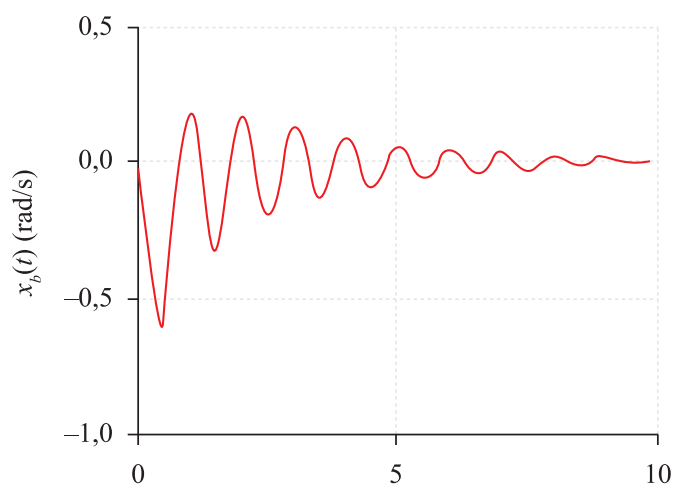

Largura de pulso

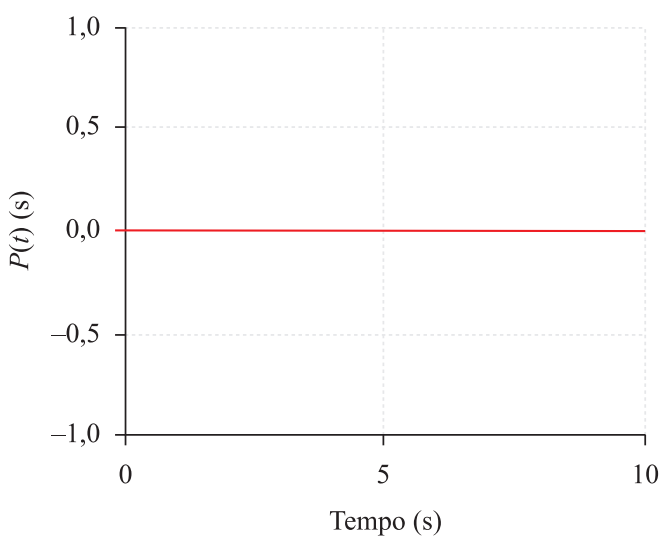

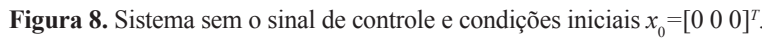
Figure 8. System without the control sign and initial condition $x_{0}=\left[\begin{array}{lll}0 & 0 & 0\end{array}\right]^{T}$.

o que era o esperado. Na Figura 6 verifica-se também que, em regime permanente, o sistema convergiu para o ponto de equilíbrio desejado, $0,52 \mathrm{rad}\left(30^{\circ}\right)$.

A Figura 7 apresenta os resultados obtidos na simulação do sistema controlado, mostrando o comportamento das variáveis posição angular e velocidade angular da articulação do joelho, e o torque produzido pelo estímulo elétrico no músculo quadríceps. O último gráfico da Figura 7 refere-se à largura de pulso enviada ao eletroestimulador. $\mathrm{Na}$ Figura 7 definem-se as variáveis $x_{a}=\theta_{v}, x_{b}=\dot{\theta}_{v}, x_{c}=M_{a}$, e o ponto de operação é dado por: $\theta_{v 0}=30^{\circ}(\pi / 6 \mathrm{rad})$, $\dot{\theta}_{v 0}=0 \mathrm{rad} / \mathrm{s}, M_{a 0}=4,6068 \mathrm{~N} \cdot \mathrm{m}$ e $P_{0}$ é igual a $P_{0}=M_{a 0} / G=1,0839 \times 10^{-4} \mathrm{~s}$. Após o transitório do sistema, ao variar o ângulo da articulação de $0^{\circ}$ a $30^{\circ}$, observa-se, em regime permanente, os seguintes parâmetros: posição $x_{a}=30^{\circ}$; velocidade angular $x_{b}=0 \mathrm{rad} / \mathrm{s}$; torque provocado pelo estímulo elétrico $M_{a}=4,6068 \mathrm{~N} \cdot \mathrm{m}$; e largura do pulso aplicado pelo neuroestimulador $P=1,0839 \cdot 10^{-4} \mathrm{~s}$.

A Figura 8 mostra o comportamento do sistema, sem a lei de controle aplicada, ou seja, no momento em que a largura de pulso torna-se nula, na posição de $30^{\circ}$. A perna volta ao repouso pela ação da gravidade. Deste modo $\theta_{v 0}=30^{\circ}(\pi / 6 \mathrm{rad}), \dot{\theta}_{v 0}=0 \mathrm{rad} / \mathrm{s}$, $M_{a 0}=4,6068 \mathrm{~N} \cdot \mathrm{m}$. Ao se retirar o sinal do músculo quadríceps, tem-se:

$$
\begin{gathered}
{\left[\Delta \theta_{v}, \Delta \dot{\theta}_{v}, \Delta M_{a}\right]=\left[\theta_{v}-\theta_{v 0}, \dot{\theta}_{v}-\dot{\theta}_{v 0}, M_{a}-M_{a 0}\right]=} \\
{\left[\pi / 6-\theta_{v 0}, 0-\dot{\theta}_{v 0}, 4,6068-M_{a 0}\right]=[0,0,0]}
\end{gathered}
$$

sendo estas as condições iniciais do sistema de retorno, saindo deste ponto e voltando para as condições de origem, $\left[0-\theta_{v 0}, 0-\dot{\theta}_{v 0}, 0-M_{a 0}\right]=[-\pi / 6,0,-4,6068]$.

\section{Discussão}

O uso de acelerômetros, fixados na perna, não promove o mesmo desconforto do eletrogoniômetro "amarrado", em parte no quadríceps, em parte na panturrilha (Winter, 2005). A literatura mostra que os sensores acelerométricos vêm sendo muito utilizados na detecção de movimento, e podem fornecer sinais precisos em qualquer direção desejada. Em Slaven et al. (2006) foram feitos vários testes com acelerômetros visando avaliar o nível de confiança dos dados fornecidos por 
estes sensores. Slaven et al. (2006) mostram que os acelerômetros apresentam ótimos resultados quando utilizados de forma adequada, e, além disso, a literatura também mostra que medidas de velocidade podem ser obtidas (com precisão) a partir da aceleração (Abdelaziz e Valášek, 2004). Em Chen et al. (1997) e Davoodi e Adrews (1998), o controle fuzzy foi empregado para controlar os movimentos cíclicos através da FES em pacientes paraplégicos. O controle fuzzy estudado em Chen et al. (1997) e Davoodi e Adrews (1998) é baseado no modelo fuzzy de King e Mandani (1977), que utiliza funções de pertinência padronizadas e ajustadas pelo projetista. Neste trabalho, o método de controle aborda uma estrutura diferente, ou seja, o sistema de controle utilizado é baseado no modelo fuzzy T-S, com funções de pertinência que dependem das não-linearidades da planta. Considerando estes fatores, verifica-se que a técnica de modelos fuzzy T-S por realimentação derivativa proposta neste trabalho é viável e de aplicação inédita, e apresenta bons resultados através das simulações. Mostra-se, com o rigor do projeto de sistemas não-lineares utilizando funções de Lyapunov, a possibilidade de unir a confiabilidade dos acelerômetros com as técnicas de projeto metódicas utilizando modelos fuzzy T-S. $\mathrm{O}$ resultado do estudo mostra que o método de controle proposto é viável. Existem vários temas para estudos futuros neste assunto, por exemplo, a robustez considerando incertezas nos parâmetros da planta (descrita na Equação 1) e a implementação do sistema de controle apresentado.

\section{Conclusão}

O controle da posição da perna de pacientes paraplégicos com eletroestimulação aplicada ao músculo quadríceps proposto, utilizando acelerômetros fixados na perna, é viável. Este artigo aborda esse problema pela primeira vez, considerando a planta não-linear descrita por modelos fuzzy T-S e realimentação da derivada do vetor de estado da planta. Simulações digitais utilizando o software MATLAB ${ }^{\circledR}$ confirmaram a eficiência do método proposto no controle da posição da perna de um paciente paraplégico, com o ângulo da articulação do joelho variando no intervalo de $0^{\circ}$ a $30^{\circ}$. O sistema de controle não-linear projetado considerou todas as não-linearidades da planta e satisfez as restrições de projeto (estabilidade segundo Lyapunov, taxa de decaimento e restrição no sinal da entrada), mostrando-se um método eficiente com aspectos de projeto bem definidos. Portanto, o objetivo do trabalho de mostrar a eficiência do método com uso de acelerômetros e técnica de controle não-linear para movimentar a perna de um paciente paraplégico, em malha fechada com ângulo da articulação do joelho, foi alcançado na simulação. Outros aspectos estão sendo levados em consideração para a implementação em hardware do sistema proposto, por exemplo, a robustez com relação às variações dos parâmetros do modelo matemático da junção do joelho do paciente.

\section{Agradecimentos}

Os autores agradecem à FAPESP, CNPq e CAPES pelo apoio financeiro recebido para realizar esta pesquisa.

\section{Referências}

Abdelaziz THS, Valášek M. Pole-placement for SISO linear systems by state-derivative feedback. IEE Proceedings Control Theory and Applications. 2004; 151(4):377-85. http://dx.doi.org/10.1049/ip-cta:20040660

Assunção E, Teixeira MCM, Faria FA, Silva NAP, Cardim R. Robust state-derivative feedback LMI-based designs for multivariable linear systems. International Journal of Control. 2007; 80(8):1260-70.

Boyd S, Ghaoui L, Feron E, Balakrishnan V. Linear matrix inequalities in systems and control theory. Philadelphia: Society for Industrial and Applied Mathematics; 1994

Cardim R, Teixeira MCM, Assunção E, Covacic MR. Design of state-derivative feedback controllers using a state feedback control design. In: 3th IFAC Symposium on System, Structure and Control, 2007: Proceedings of the IFAC SSSC2007. Iguassu Falls, 2007b. Article 135, 6 p.

Cardim R, Teixeira MCM, Assunção E, Faria FA. Control designs for linear systems using state-derivative feedback. In: Husek P, editor. Systems, Structure and Control. Vienna: InTech; 2008. p. 1-28.

Cardim R, Teixeira MCM, Assunção E, Faria FA, Covacic MR. Controle de um levitador magnético utilizando modelos fuzzy e derivada de estados da planta. In: VIII Simpósio Brasileiro de Automação Inteligente - SBAI, 2007: Anais do SBAI2007. Florianópolis, 2007a. Artigo 30693, 6 p.

Chen JJJ, Yu NY, Huang DG, Ann BT, Chang GC. Applying fuzzy logic to control cycling movement induced by functional electrical stimulation. IEEE Transactions on Rehabilitation Engineering. 1997; 5(2):158-69. PMid:9184902. http://dx.doi.org/10.1109/86.593285

Crago PE, Mortimer JT, Peckham PH. Closed-loop control of force during electrical stimulation of muscle. IEEE Transactions on Biomedical Engineering. 1980; 27(6):306-12. PMid:7390527. http://dx.doi.org/10.1109/TBME.1980.326738

Davoodi R, Adrews B. Computer simulation of FES standing up in paraplegia: A self-adaptive fuzzy controller with reinforcement learning. IEEE Transactions on Rehabilitation Engineering. 1998; 6(2):151-61. PMid:9631322. http://dx.doi.org/10.1109/86.681180

Dejnabadi H, Jolles BM, Aminian K. A new approach to accurate measurement of uniaxial joint angles based on a combination of accelerometers and 
gyroscopes. IEEE Transactions on Biomedical Engineering. 2005; 52(8):1478-84. PMid:16119244. http://dx.doi.org/10.1109/TBME.2005.851475

Dorf RC, Bishop RH. Sistemas de controle modernos. 8th ed. Rio de Janeiro: LTC; 2001.

Faria FA, Assunção E, Teixeira MCM. Realimentação da derivada dos estados em sistemas multivariáveis lineares usando LMIs. SBA Controle \& Automação. 2009a; 20(1):83-93.

Faria FA, Assunção E, Teixeira MCM, Cardim R, Silva NAP. Robust state-derivative pole placement LMI-based designs for linear systems. International Journal of Control. 2009b; 82(1):1-12. http://dx.doi.org/10.1080/00207170801942188

Ferrarin M, Pedotti A. The relationship between electrical stimulus and joint torque: A dynamic model. IEEE Transactions on Rehabilitation Engineering. 2000; 8(3):342-52. PMid:11001514. http://dx.doi.org/10.1109/86.867876

Franken HM, Veltink PH, Tijsmans R, Nijmeijer H, Boom HBK. Identification of passive knee joint and shank dynamics in paraplegics using quadriceps stimulation. IEEE Transactions on Rehabilitation Engineering. 1993; 1(3):154-64. http://dx.doi.org/10.1109/86.279264

Gahinet P, Nemirovski A, Laub AJ, Chilali M. LMI control toolbox for use with Matlab. The Math Works Inc. 1995.

Gaino R. Controle de movimentos de pacientes paraplégicos utilizando modelos Fuzzy Takagi-Sugeno [tese]. Ilha Solteira: Universidade Estadual Paulista; 2009. 179 p.

Hill AV. The heat of shortening and the dynamic constants of muscle. Proceedings of the Royal Society of London, Series B-Biological Sciences. 1938; 126(843):136-95. http://dx.doi.org/10.1098/rspb.1938.0050

King PJ, Mamdani EH. The application of fuzzy control systems to industrial process. Automática. 1977; 13(3):235-42.

Naves ELM. Modelagem e simulação do controle da postura ereta humana quase-estática com reflexos neuromusculares [tese]. Uberlândia: Universidade Federal de Uberlândia; 2006. 187 p.
Ogata K. Engenharia de controle moderno. 4th ed. New York: Prentice Hall; 2003.

Riener R, Fuhr T. Patient-driven control of FES-supported standing up: A simulation study. IEEE Transactions on Rehabilitation Engineering. 1998; 6(2):113-24. PMid:9631319. http://dx.doi.org/10.1109/86.681177

Slaven JE, Andrew ME, Violanti JM, Burchfiel CM, Vila BJ. A statistical test to determine the quality of accelerometer data. Physiological Measurement. 2006; 27(4):41323. PMid:16537982. http://dx.doi.org/10.1088/0967$3334 / 27 / 4 / 007$

Takagi T, Sugeno M. Fuzzy identification of systems and its applications to modeling and control. IEEE Transactions on Systems, Man and Cybernetics. 1985; 15(1):116-32.

Tanaka K, Ikeda T, Wang HO. Fuzzy regulators and fuzzy observers: Relaxed stability conditions and LMI-based designs. IEEE Transactions on Fuzzy Systems. 1998; 6(2):250-65. http://dx.doi.org/10.1109/91.669023

Taniguchi T, Tanaka K, Ohtake H, Wang HO. Model construction, rule reduction, and robust compensation for generalized form of Takagi-Sugeno fuzzy systems. IEEE Transactions on Fuzzy Systems. 2001; 9(4):525-37. http://dx.doi.org/10.1109/91.940966

Teixeira MCM, Deaecto GS, Gaino R, Assunção E, Carvalho AA, Farias UC. Design of a fuzzy Takagi-Sugeno controller to vary the joint knee angle of paraplegic patients. Lectures Notes in Computer Science. 2006a; 4234(3):118-26. http://dx.doi.org/10.1007/11893295_14

Teixeira MCM, Deaecto GS, Gaino R, Assunção E, Carvalho AA, Farias UC. Projeto de um controlador fuzzy TakagiSugeno para variar o ângulo da articulação do joelho. In: XVI Congresso Brasileiro de Automática, 2006:. Anais do CBA2006. Salvador, 2006b. p. 2287-92.

Teixeira MCM, Żak SH. Stabilizing controller design for uncertain nonlinear systems using fuzzy models. IEEE Transactions on Fuzzy Systems. 1999; 7(2):133-42. http://dx.doi.org/10.1109/91.755395

Winter DA. Biomechanics and motor control of human movement. New York: John Wiley Sons Inc; 2005.

\footnotetext{
Autores

Ruberlei Gaino, Márcio Roberto Covacic

Departamento de Engenharia Elétrica, Universidade Estadual de Londrina - UEL,

Rod. Celso Garcia Cid, Km 380, CEP 86051-990, Londrina, PR, Brasil

Marcelo Carvalho Minhoto Teixeira, Aparecido Augusto de Carvalho, Edvaldo Assunção, Rodrigo Cardim, Marcelo Augusto Assunção Sanches

Departamento de Engenharia Elétrica, Faculdade de Engenharia de Ilha Solteira - UNESP,

Av. José Carlos Rossi, 1370, CEP 15385-000, Ilha Solteira, SP, Brasil
} 